8-2009

\title{
RandomCast: An Energy-Efficient Communication Scheme for Mobile Ad Hoc Networks
}

Sunho Lim

South Dakota State University, sunho.lim@sdstate.edu

Chansu Yu

Cleveland State University, c.yu91@csuohio.edu

Chita R. Das

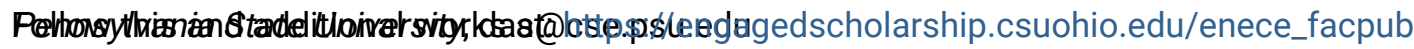

Part of the Power and Energy Commons, and the Systems and Communications Commons How does access to this work benefit you? Let us know!

\section{Publisher's Statement}

(C) 2009 IEEE. Personal use of this material is permitted. Permission from IEEE must be obtained for all other uses, in any current or future media, including reprinting/republishing this material for advertising or promotional purposes, creating new collective works, for resale or redistribution to servers or lists, or reuse of any copyrighted component of this work in other works.

\section{Original Citation}

Sunho, L., Chansu, Y., \& Das, C. R. (August 01, 2009). RandomCast: an energy-efficient communication scheme for mobile ad hoc networks. IEEE Transactions on Mobile Computing, 8, 8, 1039-1051.

\section{Repository Citation}

Lim, Sunho; Yu, Chansu; and Das, Chita R., "RandomCast: An Energy-Efficient Communication Scheme for Mobile Ad Hoc Networks" (2009). Electrical Engineering \& Computer Science Faculty Publications. 89.

https://engagedscholarship.csuohio.edu/enece_facpub/89

This Article is brought to you for free and open access by the Electrical Engineering \& Computer Science Department at EngagedScholarship@CSU. It has been accepted for inclusion in Electrical Engineering \& Computer Science Faculty Publications by an authorized administrator of EngagedScholarship@CSU. For more information, please contact library.es@csuohio.edu. 


\title{
RandomCast: An Energy-Efficient Communication Scheme for Mobile Ad Hoc Networks
}

\author{
Sunho Lim, Member, IEEE, Chansu Yu, Senior Member, IEEE, and Chita R. Das, Fellow, IEEE
}

\begin{abstract}
In mobile ad hoc networks (MANETs), every node overhears every data transmission occurring in its vicinity and thus, consumes energy unnecessarily. However, since some MANET routing protocols such as Dynamic Source Routing (DSR) collect route information via overhearing, they would suffer if they are used in combination with 802.11 PSM. Allowing no overhearing may critically deteriorate the performance of the underlying routing protocol, while unconditional overhearing may offset the advantage of using PSM. This paper proposes a new communication mechanism, called RandomCast, via which a sender can specify the desired level of overhearing, making a prudent balance between energy and routing performance. In addition, it reduces redundant rebroadcasts for a broadcast packet, and thus, saves more energy. Extensive simulation using ns-2 shows that RandomCast is highly energy-efficient compared to conventional 802.11 as well as 802.11 PSM-based schemes, in terms of total energy consumption, energy goodput, and energy balance.
\end{abstract}

Index Terms-Energy balance, energy efficiency, mobile ad hoc networks, network lifetime, overhearing, power saving mechanism.

\section{INTRODUCTION}

major concern in mobile ad hoc networks (MANETs) is A energy conservation due to the limited lifetime of mobile devices. Since wireless communication could be responsible for more than half of total energy consumption [16], a great deal of effort has been devoted to develop energy-aware network protocols such as Power-aware routing [5], [24], [30], [31] and transmit power control (TPC)-based algorithms [3], [8], [13], [15]. Essentially, they have concentrated on reducing energy spent for active communication activities.

However, wireless radios still consume energy during the period of inactivity. In fact, idling listening usually accounts for a larger part of the total energy consumption because radios remain inactive for a longer duration [22]. Therefore, many radio hardware support low-power sleep state, during which substantially low energy is consumed but no communication is allowed [14]. For instance, Lucent's WaveLAN-II consumes 1.15 Watt and 0.045 Watt in the idle listening and low-power sleep state, respectively [14]. More than 25 times smaller energy cost clearly presents the benefit of using the low-power sleep state.

IEEE 802.11 exploits this hardware capability to support the Power Saving Mechanism (PSM) in its medium access control (MAC) layer specification [2]. Each radio can be in one of two power management modes: active mode (AM) or power save (PS) mode. A device in AM stays awake all the time. It can communicate at any moment but wastes energy during idling. A device in PS mode periodically wakes up during the packet advertisement period, called Ad hoc (or Announcement) Traffic Indication Message (ATIM) window, to see if it has any data to receive. It puts itself into the lowpower sleep state during the subsequent data transmission period if it is not addressed, but stays awake otherwise to receive an advertised packet. However, 802.11 PSM is originally designed for single-hop wireless LANs and further research is required to efficiently use it in a multihop MANET.

The main goal of this paper is to make the 802.11 PSM applicable in a MANET with Dynamic Source Routing (DSR) [12] and to achieve an additional energy saving by identifying and eliminating unnecessary communication activities. More specifically, this paper has been motivated by the following two observations. First, a main trouble in integrating the DSR protocol with 802.11 PSM comes from unnecessary or unintended overhearing. Overhearing improves the routing efficiency in DSR by eavesdropping other communications to gather route information but it spends a significant amount of energy. Second, it is important to note that most of network layer solutions developed for MANETs including DSR depend on broadcast flood of control packets. Unconditional forwarding of broadcast packets is wasteful and even harmful because it generates many redundant rebroadcasts. This paper proposes a message overhearing and forwarding mechanism, called RandomCast, which makes a judicious balance between energy and network performance. In RandomCast, a node may decide not to overhear (a unicast message) and not to forward (a 
broadcast message) when it receives an advertisement during an ATIM window, thereby reducing the energy cost without deteriorating the network performance.

Key contributions of this paper are threefold: 1) It presents the RandomCast protocol that is designed to employ the IEEE 802.11 PSM in multihop MANETs. Unlike previous approaches, where nodes need to switch between AM and PS mode, they consistently operate in the PS mode in RandomCast. This has not been studied elsewhere in the literature to the best of authors' knowledge. 2) In RandomCast, a transmitter can specify the desired level of overhearing to strike a balance between energy and throughput. More importantly, it helps avoid the semantic discrepancy found in most of MANET routing protocols. For example, in DSR, when a node transmits a unicast packet, it in fact expects that all of its neighbors overhear it as if it is a broadcast packet. This is not the case in the proposed RandomCast protocol. 3) Compared to our earlier work [19], this paper shows that the problem of unconditional or unnecessary forwarding of broadcast packets can also be taken care of in the RandomCast framework.

The performance of the proposed RandomCast scheme is evaluated using the ns-2 network simulator [1] in comparison to 802.11, 802.11 PSM, and On-Demand Power Management (ODPM) [32]. ODPM is one of the most energy-efficient MAC schemes developed for MANETs and is discussed in detail in Section 2.2. According to the simulation results, the proposed algorithm reduces the energy consumption as much as 50 percent and 31 percent compared to the original IEEE 802.11 PSM and ODPM, respectively. On the other hand, network performance such as its packet delivery ratio (PDR) could be at a disadvantage with RandomCast because nodes are not able to transmit or receive packets when they are in sleep state. In order to examine the performance trade-offs, we measure a combined metric, called energy goodput (Kbytes/Joule), which is defined as the number of bytes delivered per unit energy. RandomCast achieves as much as 64 percent and 63 percent higher energy goodput than 802.11 PSM and ODPM, respectively, which exhibits the overall benefit of RandomCast.

The rest of the paper is structured as follows: Section 2 presents the background information on the DSR routing protocol and IEEE 802.11 PSM. Section 3 presents the proposed RandomCast protocol and its integration with DSR. Section 4 is devoted to extensive performance analysis. Section 5 discusses the issue of packet latency, which is a demerit of 802.11 PSM and its variants such as RandomCast. Section 6 draws conclusions and presents future directions of this study.

\section{BACKGROUND}

We assume that mobile nodes employ the IEEE 802.11 PSM for energy-efficient use of the shared medium and DSR for discovering and maintaining routing paths. Section 2.1 summarizes the DSR routing protocol. It also discusses the effect of overhearing in DSR and argues that unconditional overhearing and rebroadcast is the main reason behind

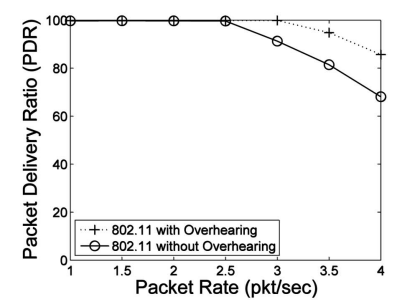

(a)

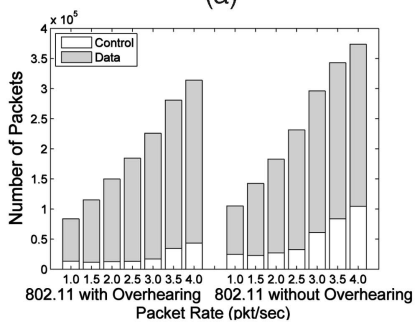

(c)

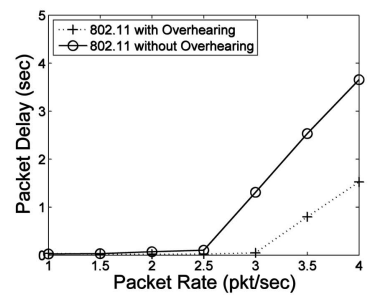

(b)

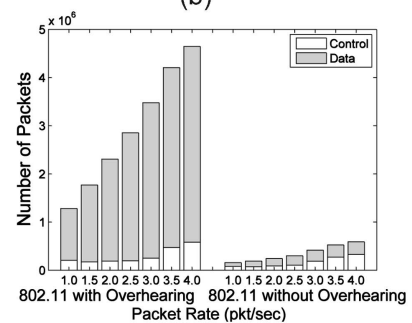

(d)
Fig. 1. Effect of overhearing. (a) Packet delivery ratio (PDR), (b) packet delay, (c) number of packets transmitted (traffic implication), and (d) number of packets received or overheard (energy implication). (50 nodes in $300^{-} \times 1500-\mathrm{m}^{2}$, 30 CBR streams generated by 21 nodes, pause time of 100 seconds, and the maximum node speed of $5 \mathrm{~m} / \mathrm{s}$. Note that the scale in (d) is 10 times larger than in (c).)

energy inefficiency. Section 2.2 explains 802.11 PSM and previous research work on its use in single-hop and multihop networks.

\subsection{DSR Routing Protocol}

\subsubsection{Route Discovery and Maintenance}

When a node has a data packet to send but does not know the routing path to the destination, it initiates the route discovery procedure by broadcasting a control packet, called route request (RREQ). When an RREQ reaches the destination, it prepares another control packet, called route reply (RREP), and replies back to the source with the complete route information. Upon receiving an RREP, the source saves the route information in its local memory, called route cache, for later uses. Since nodes move randomly in a MANET, link errors occur and a route information that includes a broken link becomes obsolete. When a node detects a link error during its data transmission, it sends another control packet, called route error (RERR), to the source and deletes the stale route from its route cache. Overhearing improves the network performance by allowing nodes to collect more route information. Nodes in the vicinity of a transmitter would learn about the path to the destination via overhearing.

\subsubsection{Effect of Overhearing}

To better understand the effect of overhearing in terms of routing performance and its implication on energy consumption, Fig. 1 compares 802.11 with and without overhearing $^{1}$ under the simulation scenario detailed in Section 4.1. Figs. $1 \mathrm{a}$ and $1 \mathrm{~b}$ compare the two in terms of packet delivery ratio (PDR) and packet delay, respectively. As can be inferred from the figure, overhearing improves

1. In the DSR implementation in ns-2 (version 2.30), overhearing can be simply disabled by modifying the constant "dsragent_use_tap" to false. 
the network performance, pronounced more at higher traffic condition. To identify the cause of the performance difference, the number of packets transmitted and that received/overheard is compared in Figs. $1 \mathrm{c}$ and 1d, respectively. In both figures, data packets are dominant. However, the number of control packets (RREQ, RREP, and RERR) increases when packet rate increases as shown in the figure. In Fig. 1c, 802.11 without overhearing results in more control packets and a slightly larger number of data packets than with overhearing. Total traffic in the network is not significantly different as in Fig. 1c.

However, the number of packets received or overheard shows the opposite trend and the gap is quite significant as shown in Fig. 1d. Although less traffic is generated, nodes receive or overhear more packets when overhearing is enabled. Note that energy consumption of a node is usually dominated by the packets received/overheard because they are much more than those transmitted. Note that the scale in Fig. 1d is 10 times larger than in Fig. 1c. In short, overhearing increases traffic (and energy consumption) but improves network performance. It is, therefore, important to know how to make a prudent trade-off between the two and how to control the level of overhearing.

\subsubsection{Unconditional Overhearing and Stale Route Problem in DSR}

In addition to the energy inefficiency, overhearing brings in several undesirable consequences. For example, it could aggravate the stale route problem, the main cause of which is node mobility. When nodes move, wireless links break and an upstream node propagates an RERR packet to evict stale route information from route caches of the nodes along the path. However, since link error information (or RERR) is not propagated "fast and wide" [20], route caches often contain stale routes for an extended period of time.

Now, overhearing could make the situation worse. This is because DSR generates more than one RREP packets for a route discovery to offer alternative routes in addition to the primary one. While the primary route is checked for its validity during the communication between the source and the destination, alternative routes may remain in route cache unchecked even after they become stale. This applies not only for the nodes along the alternative routes, but also for all their neighbors because they learned and kept them via unconditional overhearing.

\subsubsection{Unconditional Overhearing and Semantic Discrepancy in DSR}

Every node in DSR aggressively collects route information via overhearing but it introduces a semantic discrepancy as discussed in Section 1. A unicast packet (data, RREP, or RERR) is intended for the designated receiver only, but the transmitter in fact wishes that other nodes in the proximity overhear it. However, when nodes employ 802.11 PSM, they do not wake up to overhear unintended packets, disrupting the normal operation of DSR. In the proposed RandomCast algorithm, a desired level of overhearing can be specified for each packet and thus, every communication is semantically consistent.

\subsubsection{Unconditional Rebroadcast in DSR}

Another source of excessive energy consumption in DSR is unconditional rebroadcast. An RREQ control packet is meant to every other node in the network, and thus, each node is supposed to rebroadcast it whenever it receives one. However, in a dense network, some rebroadcasts of RREQs are redundant while increasing the network traffic as well as wasting energy resource for transmitting and receiving the rebroadcasts. This may not be a big trouble when RREQs are rarely generated as in a network with no or less mobility. On the other hand, when node mobility is high, there will be more link breaks, causing more broadcast packets (RREQs) as well as their flooding in the network.

This is a well-researched problem, known as broadcast storm [25]. Recently, there has been an active research on a probabilistic protocol, called gossiping [4], [9], [17], [21], which addresses this problem. A node decides whether or not to rebroadcast a packet based on gossiping probability. The main goal is to minimize the number of routing packets such as RREQs without degrading the network performance. We will show in Section 3 that the proposed RandomCast protocol implements the idea of gossiping in the same way as it does for unconditional overhearing.

\subsection{IEEE 802.11 Power Saving Mechanism (PSM)}

According to the IEEE 802.11 standard [2], there are two medium access methods depending on the existence of an access point (AP). They are referred to as Distributed Coordination Function (DCF) and Point Coordination Function (PCF). The DCF uses a contention algorithm based on the principle of Carrier Sense Multiple Access with Collision Avoidance (CSMA/CA) and delay, known as InterFrame Space (IFS). The PCF is an optional access method implemented on top of DCF and provides a contentionfree service coordinated by an AP.

\subsection{PSM in Single-Hop Networks}

Power saving in PCF is achieved by the coordination of the AP. As discussed in Section 1, each node operates either in AM or PS mode. With PCF, the AP operates in AM and all other mobile nodes operate in PS mode. The AP periodically sends a beacon for synchronizing mobile nodes in its neighborhood. The beacon includes Traffic Indication Map (TIM), which is a bitmap vector to indicate the traffic and the corresponding receiver. If a node is specified as a receiver in the TIM, it remains awaken to receive a packet during the following data transmission period. It switches off its radio subsystem otherwise.

In the DCF, power saving is more difficult to achieve. In the absence of an AP, nodes in the PS mode should synchronize among themselves in a distributed way [11], [26]. In addition, a beacon does not contain the TIM, and each sender should advertise its own packet by transmitting an Ad hoc TIM (ATIM) frame during the packet advertisement period, called ATIM window. Each packet is buffered at the sender and is directly transmitted to the receiver during the following data transmission period.

Fig. 2 shows the PSM protocol in the DCF with an example mobile network of five nodes, $S_{1}, R_{1}, S_{2}, R_{2}$, and $R_{3}$. In Fig. 2a, node $S_{1}$ - has a unicast packet for node $R_{1}$-and 


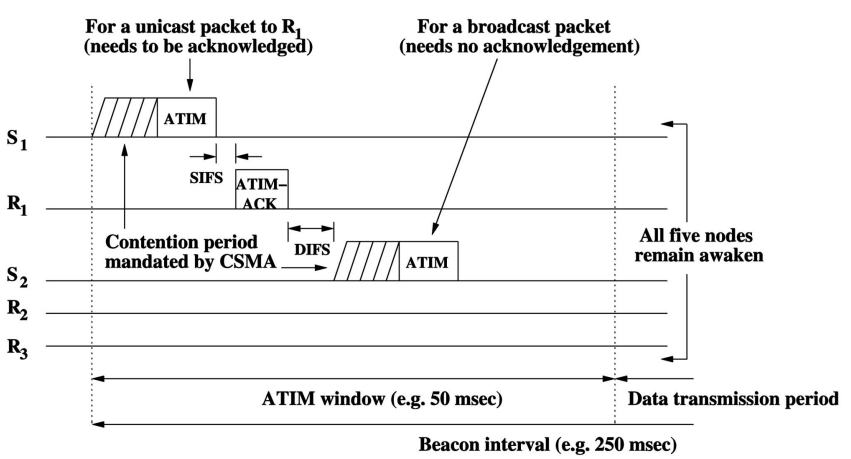

(a)

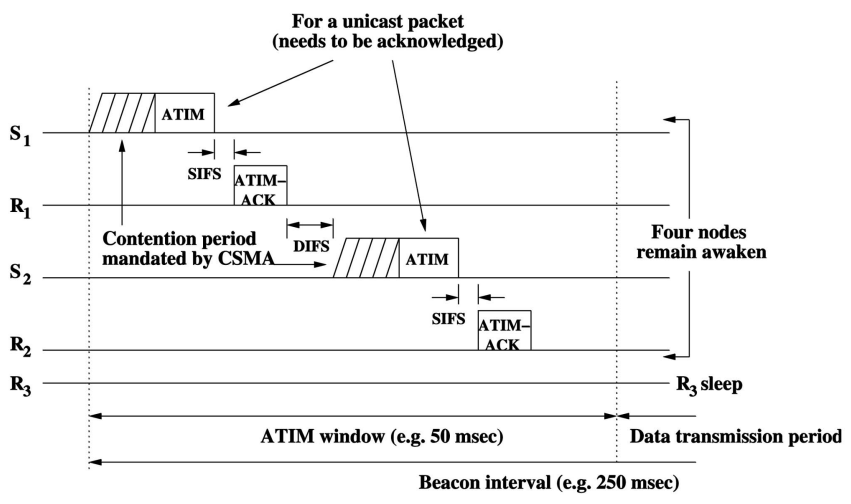

(b)

Fig. 2. IEEE 802.11 PSM (SIFS: Short IFS and DIFS: DCF IFS). (a) One unicast and one broadcast packet (all five nodes remain awaken during the entire beacon interval) and (b) two unicast packets (four nodes remain awaken but node $R_{3}$-sleeps during the data transmission period).

node $S_{2}$-has a broadcast packet. They advertise them during the ATIM window as shown in the figure. Note that nodes $S_{1}$ and $S_{2}$-compete against each other using the CSMA/CA principle for transmitting the ATIM frames. Node $S_{1}$-needs an acknowledgment (ATIM-ACK) from node $R_{1}$-but node $S_{2}$-does not. In this scenario, all five nodes remain awaken during the data transmission period in order to receive the unicast and/or the broadcast packet. Consider another example in Fig. $2 \mathrm{~b}$. Here, nodes $S_{1}$ - and $S_{2}$ have a unicast packet to $R_{1}$ and $R_{2}$, respectively, and thus, nodes $S_{1}, R_{1}$, $S_{2}$, and $R_{2}$ must be awaken. However, node $R_{3}$-can switch to the low-power sleep state immediately after the ATIM window because it does not have any packet to receive. It is noted that node $R_{3}$ - should remain awaken if it needs to overhear unconditionally.

\subsection{PSM in Multihop Networks}

Note that the aforementioned scenarios assume that every node is within every other's radio transmission range. Thus, they are not directly applicable in multihop mobile networks. Recently, a number of research groups have studied how to utilize the PSM in multihop networks. SPAN [6] mandates a set of nodes to be in AM, while the rest of the nodes stay in the PS mode. AM nodes offer the routing backbone so that any neighboring node can transmit a packet to one of them without waiting for the next beacon interval. A drawback of this scheme is that it usually results in more AM nodes than necessary and degenerates to all AM-node situation when the network is sparse. More importantly, it does not take the routing overhead into account because it uses geographic routing and assumes that location information is available for free. This is neither realistic nor compatible for use with DSR as pointed out in [18], [28].

Zheng and Kravets suggested a similar approach, called On-Demand Power Management (ODPM) [32], in which a node switches between the AM and PS mode based on communication events and event-induced time-out values. For example, when a node receives an RREP packet, it is better to stay in AM for an extended period of time (RREP time-out) because it will most probably need to forward data packets in the near future. However, this scheme asks for each node to switch between the AM and PS mode frequently, which may incur non-negligible overhead. Moreover, each node needs to know and maintain the power management mode of its neighbors. This may not be trivial as it requires either an additional energy cost or an extended packet delay if the information is not accurate. Also, its performance greatly depends on time-out values, which need fine tuning with the underlying routing protocol as well as traffic conditions. For example, consider that a node stays in AM for five consecutive beacon intervals upon receiving a data packet (Data time-out) as suggested in [32]. If data traffic is infrequent, say once every six beacon intervals, the node stays in AM for five intervals without receiving any further data packets and switches to a low-power sleep state. It receives the next data packet while operating in the PS mode, and thus, decides again to stay awaken for another five intervals. Packet delay is not improved but it consumes more energy than unmodified 802.11 PSM.

Alternatively, a probabilistic decision on AM or PS mode has been studied in recent years, in which a backbone network consisting of AM nodes is constructed as in SPAN. However, unlike SPAN, the number of AM nodes is reduced based on heuristics. In $\mathrm{Li}$ and $\mathrm{Li}^{\prime} \mathrm{s}$ recent paper [18], each node makes an AM-node (backbone) decision based on the number of neighbors; i.e., the backbone probability $(P)$ is inversely proportional to the number of neighbors (say, $n$ ). This is based on the observation that having more neighbors usually means more redundancy in terms of connectivity. The backbone probability is then adjusted based on the average number of neighbors of its neighbors (say, $\bar{n}$ ). In other words, when a node has more neighbors than its neighbors, its backbone probability is increased because it can help reduce the number of AM nodes by electing itself as an AM node, i.e., $P \psi=\frac{c n}{\bar{n}^{2}}$, where $c$ is a tunable constant [18].

Traffic-Informed Topology-Adaptive Network (TITAN) is another probabilistic algorithm that improves over ODPM. It favors AM nodes when selecting routing paths at the network layer [23]. It can be easily accomplished when PS nodes delay forwarding RREQ packets. Discovered routes could be a long way around compared to the shortest ones, but they utilize more AM nodes for delivering traffic. PS nodes would sleep for a longer duration than in ODPM and 


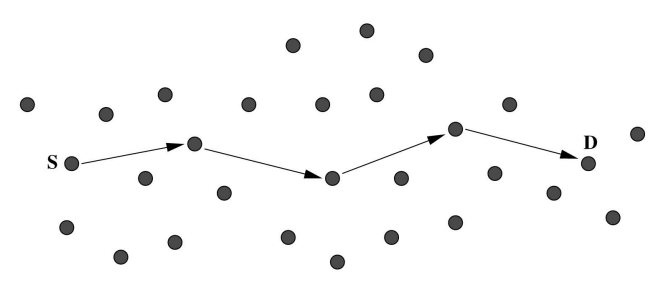

(a)

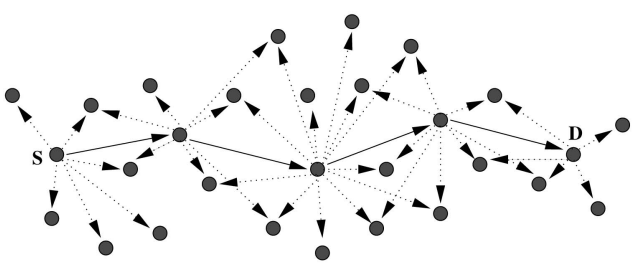

(b)

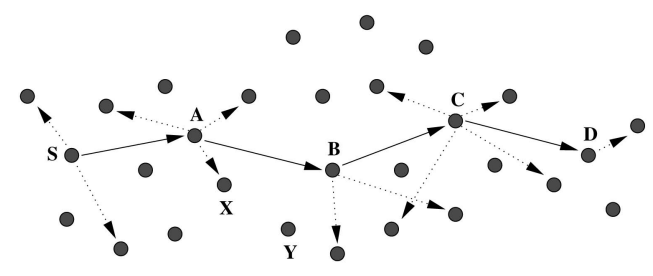

(c)

Fig. 3. Delivery of a unicast message with different overhearing mechanisms. (a) no overhearing, (b) unconditional overhearing, and (c) randomized overhearing.

save more energy. The backbone decision (AM node) depends on the number of neighbors as well as the number of neighboring AM nodes.

Our approach in this paper is different from the aforementioned schemes in that every node operates in PS mode and is not required to switch between AM and PS mode. This means that any node won't fall in a potential danger to be an AM node for an extended period of time and die earlier than others. This could affect the network lifetime too. RandomCast not only reduces the overall energy consumption but also improves the energy balance among the nodes leading to a longer network lifetime.

\section{Randomized OVERHEARING AND ForWARDING USING RANDOMCAST}

This section describes the proposed Random Cast protocol. It is designed to improve energy performance by controlling the level of overhearing and forwarding without a significant impact on network performance. Compared to the algorithms presented in Section 2.2, the proposed scheme assumes that mobile nodes employ 802.11 PSM and consistently operate in the PS mode. Section 3.1 presents the basic idea of RandomCast and its advantages. Sections 3.2 and 3.3 discuss the RandomCast mechanism for unicast and broadcast packets, respectively. Randomization algorithm is described in Section 3.4, and Section 3.5 analyzes the trade-off between energy and the quality of route information in RandomCast. Other design issues are presented in Section 3.6.
Format of an ATIM frame (length in octets)

\begin{tabular}{|c|c|c|c|c|c|c|c|c|c|}
\hline FC & DI & DA & & SA & \multicolumn{2}{|c|}{ BSSID } & SC & Frame body & FCS \\
\hline \multicolumn{10}{|c|}{${ }_{2} \cdots_{2} \rightarrow l_{6} \quad \cdots$} \\
\hline \multicolumn{10}{|c|}{$\begin{array}{l}\text { DI: Duration/Connection ID } \\
\text { DA, SA, BSSID: Addresses of destination, source, and IBSS } \\
\text { SC: Sequence control } \\
\text { Frame body: Null for ATIM frame } \\
\text { FCS: Frame check sequence }\end{array}$} \\
\hline \multicolumn{10}{|c|}{ FC: Frame control (length in bits) } \\
\hline \begin{tabular}{|l|} 
Protocol \\
Version
\end{tabular} & Type & Subtype & $\begin{array}{l}\text { To } \\
\text { DS }\end{array}$ & $\begin{array}{c}\text { From } \\
\text { DS }\end{array}$ & \begin{tabular}{l|l|} 
More & \\
Frag & Retry \\
\end{tabular} & \begin{tabular}{|l|} 
Pwr \\
Mgt \\
\end{tabular} & \begin{tabular}{|l|} 
More \\
Data
\end{tabular} & WEPOrder & \\
\hline 2 & 2 & 4 & 1 & 1 & $\overrightarrow{1} \overrightarrow{1}$ & $\overrightarrow{1}$ & $\overrightarrow{1}$ & $\overrightarrow{1}+\overrightarrow{1}$ & \\
\hline $\begin{array}{l}\text { Type: } 00 \\
\text { Subtype: }\end{array}$ & $\begin{array}{l}0.91 \text { for } \\
101 \text { fo }\end{array}$ & $\begin{array}{l}\text { nent fram } \\
\text { IM frame } \\
\text { IM frame }\end{array}$ & such & $\begin{array}{l}\text { as ATII } \\
\text { ndition: } \\
\text { lomized }\end{array}$ & $\begin{array}{l}\text { IM frame } \\
\text { al overhearir } \\
\text { d overhearing }\end{array}$ & & & & \\
\hline
\end{tabular}

Fig. 4. ATIM frame format. (Note that RandomCast additionally defines subtypes $1101_{2}$ and $1110_{2}$ to specify randomized and no overhearing, respectively. IBSS: Independence Basic Service Set, DS: Distribution System, and WEP: Wired Equivalent Privacy).

\subsection{No, Unconditional, and Randomized Overhearing}

In RandomCast, a transmitter is able to specify the desired level of overhearing. Consider that node $S$ transmits packets to node $D \psi$ via a precomputed routing path with three intermediate nodes as shown in Fig. 3a. Only five nodes are involved in the communication and the rest would not overhear it (no overhearing). However, it is oftentimes the case that every neighbor is supposed to overhear as in DSR. This is shown in Fig. 3b (unconditional overhearing).

Randomized overhearing adds one more possibility in between the two. As shown in Fig. 3c, some of the neighbors overhear but others do not. Those that chose not to overhear will switch to a low-power sleep state during the following data transmission period, saving substantial amount of energy compared to unconditional overhearing. With respect to route information, this does not necessarily deteriorate the quality of route information due to its spatial and temporal locality of route information. Consider an example in Fig. 3c, in which nodes $A \psi$ and $B \psi$ are two intermediate nodes along the path from node $S$ to $D$. Node $B$ worwards an RREP to node $A$ w (and ultimately to node $S$ ) and later node $A$ forwards a number of data packets to node $B \psi($ and ultimately to node $D)$. Nodes $X \psi$ and $Y \psi$ are two neighbors of $A$ and $B$, and they will learn about the routing path $(S \psi \rightarrow \oplus)$ by overhearing any one of the communications between nodes $A$ yand $B$.

\subsection{RandomCast for Unicast Packets}

The RandomCast protocol enables a transmitter to choose no, unconditional, or randomized overhearing for its neighbors. It is specified in the ATIM frame and is available to its neighboring nodes during the ATIM window. For practicality, it is implemented in the context of IEEE 802.11 specification by slightly modifying the ATIM frame format as shown in Fig. 4. ${ }^{2}$ ATIM frame is a management frame (type $00_{2}$ ) and its subtype is $1001_{2}$-according to the 802.11 standard. The RandomCast protocol utilizes two unused subtypes, $1101_{2}$ and $1110_{2}$, to specify randomized and no overhearing, respectively. An ATIM frame with the original

2. Note that "PwrMgt" in FC indicates the power management mode, either AM or PS, in which the sender of the frame will stay after the current communication is successfully completed [2]. 
subtype $1001_{2}$ - is recognized as unconditional overhearing and thus conforms to the standard.

When a node (its MAC address $M A$ ) wakes up at the beginning of a beacon interval, it receives an ATIM frame for a unicast packet. The ATIM frame contains the receiver address $(D A)$ and subtype $(I D)$. The node decides whether or not to receive/overhear the advertised packet in the following data transmission period based on $D A$ and $I D$. It would remain awaken to receive it if one of the following conditions is satisfied:

1. The node is the intended destination $(D A \&-M A)$.

2. The node is not the destination but the sender wants unconditional overhearing $(D A \not \neq \mathrm{MA} A \mathrm{but}$ $\left.I D \notin \leftrightarrow 1001_{2}\right)$.

3. The node is not the destination, but the sender wants randomized overhearing, and the node randomly decides to overhear the packet $(D A \not \neq M A, I D \notin \leftarrow$ $1101_{2}$, and decides to overhear).

Now, as a transmitter, let us consider which level of overhearing is desirable for various types of unicast packets. DSR employs three control packets: RREQ, RREP, and RERR. RREQ is a broadcast, and RREP, RERR, and data are unicast packets. For each of the unicast packets, DSR uses the following overhearing mechanism:

- Randomized overhearing for RREP packets: An RREP includes the discovered route and is sent from the destination to the originator of the corresponding RREQ packet. For example, in Fig. 3c, node $D$ sends an RREP to node $S$. Unconditional overhearing of RREP is not a good idea because DSR generates a large number of RREP packets, as discussed in Section 2.1. Therefore, intermediate nodes as well as node $D$ will specify the randomized overhearing for RREP packets.

- Randomized overhearing for data packets: In DSR, every data packet includes the entire route from source to destination. Each intermediate node (e.g., nodes $A$, $B$, and $C \psi$ in Fig. 3c) as well as the source node (e.g., node $S \psi$ in Fig. 3c) will demand randomized overhearing for these packets so that neighboring nodes (e.g., nodes $X \psi$ and $Y \psi$ in Fig. 3c) can overhear them probabilistically.

- Unconditional overhearing for RERR packets: When a link (e.g., link $B-C$ in Fig. 3c) is detected broken, an upstream node (e.g., node $B$ in Fig. 3c) transmits an RERR to the source. It is better for nodes in the vicinity to overhear this message unconditionally because the stale route information must be propagated as soon and wide as possible.

\subsection{RandomCast for Broadcast Packets}

Note that the RandomCast algorithm can also be applied to broadcast packets such as RREQ to allow randomized rebroadcast as mentioned earlier. This is to avoid redundant rebroadcasts of the same packet in dense mobile networks. On the other hand, the rebroadcast decision must be made conservatively. This is because a broadcast packet may not be delivered to all nodes in the network when randomized rebroadcast is used. For example, an RREQ packet may not reach the specific destination node. For this reason, rebroadcast probability $\left(P_{F}\right)$ is set higher than overhearing probability $\left(P_{R}\right)$.

In RandomCast, when a node sends an ATIM for a broadcast packet, all of its neighbors receive the packet in the following data transmission period but probabilistically rebroadcast it. Note the difference between the randomized overhearing of a unicast packet and the randomized rebroadcast of a broadcast packet. In the former, the decision is whether to remain awake and receive the data packet after receiving an ATIM. However, in the latter, the decision is whether to rebroadcast or not. Receiving a broadcast packet is mandatory because the ultimate receiver of the broadcast packet could be in the neighborhood of the transmitter.

As in overhearing, different broadcast packets are treated differently.

- Randomized rebroadcast for RREQ packets: DSR requests a randomized rebroadcast of an RREQ packet to the MAC and the MAC forwards it probabilistically based on $P_{F}$. If the node is the source of the RREQ, it will ask the MAC to broadcast it unconditionally.

- Unconditional rebroadcast for ARP (address resolution protocol) request packets: ARP request packets are typically single-hop communication. Since the destination node is expected to exist in the transmitter's vicinity, unconditional rebroadcast must be requested to the MAC.

Note that randomized rebroadcast is more effective when node density or node mobility is high as described in Section 2.1. Note also that randomized rebroadcast of a broadcast packet is requested by an upper layer protocol (such as DSR or ARP) to the MAC as described above. The overall RandomCast algorithm is summarized in Fig. 5. More details regarding $P_{R \psi}$ and $P_{F \psi}$ are followed in the next section.

\subsection{Random Cast Probability}

A key design issue in the RandomCast implementation is randomization. Basically, each node maintains an overhearing (rebroadcast) probability, $P_{R \psi}\left(P_{F}\right)$, determined using the factors listed below. In other words, if a randomly generated number is higher than $P_{R}$, then a node decides to overhear (rebroadcast).

- Sender ID: The main objective of RandomCast is to minimize redundant overhearing. Since a node would typically propagate the same route information in consecutive packets, a neighbor can easily identify the potential redundancy based on the sender ID. For instance, when a node receives an ATIM frame with subtype $1101_{2}$, it determines to overhear it if the sender has not been heard for a while. This means that the traffic from the sender happens rarely or the node skips too many packets from the sender.

- Number of neighbors: When a node has a large number of neighbors, there potentially exists a high redundancy. For example, when a node asks for a routing path by sending an RREQ, it is possible that a neighbor offers one. 


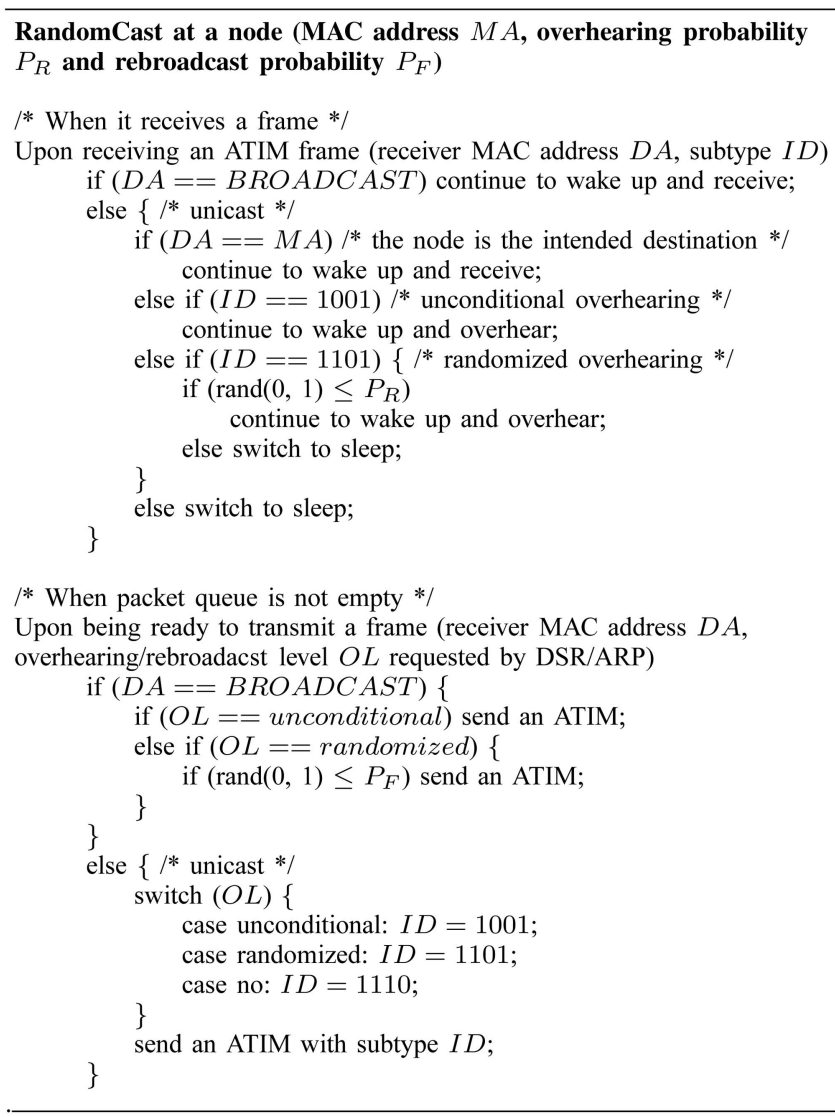

Fig. 5. The RandomCast algorithm.

- Mobility: When node mobility is high, link errors occur frequently and route information stored in route caches becomes stale easily. Therefore, it is recommended to overhear more conservatively (a higher $P_{R}$ ) but to rebroadcast more aggressively (a lower $P_{F}$ ) in this case. Each node can estimate its mobility based on connectivity changes with its neighbors.

- Remaining battery energy: This is one of the most obvious criteria that helps extend the network lifetime: less overhearing (a lower $P_{R}$ ) and less rebroadcast (a lower $P_{F}$ ) if remaining battery energy is low. However, it is necessary to take other nodes' remaining battery energy into consideration in order to achieve a balanced energy consumption.

Overhearing decision can be made based on the criteria mentioned above, but in this paper, we adopt a simple scheme using only the number of neighbors $\left(P_{R \psi}=1 / \psi\right.$ number of neighbors) to show the potential benefit of RandomCast. On the other hand, the rebroadcast probability, $P_{F}$, is based on a method introduced in Section 2.2, i.e., $P_{F \psi}=\frac{c n \psi}{\bar{n}^{2}}$, where $n u$ is the number of neighbors, $\bar{n} \psi$ is the average number of neighbors' neighbors, and cus a tunable constant (4.0 is used in this paper as suggested in [18]).

\subsection{Trade-Off between Energy and Quality of Route Information}

This section quantifies the trade-off between energy and the quality of route information in RandomCast. Let us concentrate on node $X \psi$ in Fig. $3 c$ in Section 3.1. Assume that source node $S i$ sends $k$ packets to destination node $D$.
Therefore, an intermediate node $A$ forwards $k$ packets to the next-hop node $B$, and node $X \psi$ independently decides whether or not to overhear each of the $k \psi$ packets. The following analysis estimates the probability, $P_{k}$, that node $X \psi$ or one of its direct neighbors has a route information for node $D$, given that $k$ communications toward $D$ happened in the proximity. Considering expand ring search in DSR [12], route information in its neighbors' route cache is considered readily available to node $X$.

- First, the probability $(p)$ that a neighbor of node $X$ is within the transmit range of node $A$ can be written as

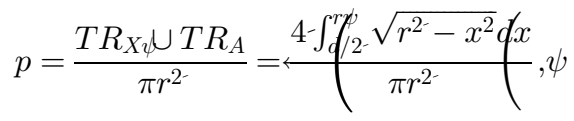

where $r \psi$ and $d \psi d$ denote the transmit range and $A-X \psi$ distance, respectively, and $T R_{X \psi}$ and $T R_{A \psi}$ denote the transmit area of node $X \psi$ and $A$, respectively [27]. Since $d \in[0, r], p$ is estimated 0.59 on the average.

- Second, let the probability $(q)$ be that at least one neighbor of node $X$ overhears a packet transmission from node $A$. Since each of nuneighbors of node $X \psi$ overhears with the probability $P_{R \psi}=\frac{1}{n}, q \psi$ can be estimated as $1-\left(1-P_{R}\right)^{p m}$.

- Therefore, the probability $\left(P_{k}\right)$ that at least one neighbor of node $X \psi$ overhears one of $k \psi$ packet transmissions from node $A \psi$ is computed as $P_{k \psi}=1-(1-q)^{k}$.

A simple calculation shows that $P_{k i}$ is higher than 95 percent when $k>\not y$ and is higher than 99 percent when $k>\boldsymbol{\psi}$ as long as $n>$ \% . In other words, in RandomCast with $P_{R \psi}=\frac{1}{n}$, the quality of route information is impacted very little in comparison to unconditional overhearing.

Now, consider the energy performance in RandomCast in comparison to the conventional case of unconditional overhearing. In RandomCast, nodes are always awake during the ATIM window (size of $a$ ) but probabilistically $\left(\frac{1}{n}\right)$ during the rest of the beacon interval (size of $b$ ). Therefore, relative energy consumption can be roughly estimated as $\frac{a+\frac{b-a}{n w}}{b \psi}$ when we count the overheard packets only. This estimate is not outrageous because overhearing is a major contributor as shown in Fig. 1. When aus $50 \mathrm{msec}$ and $b$ is $250 \mathrm{msec}$ as used throughout this paper, it becomes $\frac{n+4}{5 n *}$. For example, when $n=6$, RandomCast consumes about 33 percent of energy of unconditional overhearing. ${ }^{3}$

This analysis shows that RandomCast enhances energy performance without hurting the quality of route information obtained via overhearing.

\subsection{Other Implementation Details}

This section discusses other design issues and implementation details of RandomCast. It also discusses the implementation of ODPM [32] because it offers a reference performance in comparison to RandomCast.

3. Simulation study in the next section shows that it is $48-84$ percent in Fig. 6b. The analysis-simulation gap is due to active transmissions and receptions in addition to overhearing. Note that the gap increases as packet rate increases. 
- ARP reply: When a transmitter knows the IP address of the receiver but does not know its MAC address, it first of all sends an ARP request followed by an ARP reply from the receiver. This happens frequently during route discovery procedures. Consider that a destination receives an RREQ which includes the identities of the source as well as all intermediate nodes. However, it may not know the MAC address of the next upstream node, which may also be the case for all intermediate nodes. An ARP request is announced (during an ATIM window) and sent in a beacon interval. The corresponding ARP reply will only be announced and sent in the next beacon interval, dramatically increasing the discovery latency. It can be greatly reduced when it is allowed to send an ARP reply immediately upon receiving an ARP request [7]. Since ARP request is sent after it is announced during an ATIM window, the receiver of the ARP reply packet is most probably awake during the same data transmission period. Therefore, the ARP receiver does not have to wait for the next beacon interval to announce the ARP reply packet.

- Multiple ATIMs: A node is allowed to send more than one ATIM frame if it has more than one packet to send. Therefore, a node can send multiple packets in a beacon interval, improving the network performance.

- Neighbor information: Each node maintains information about its neighbors such as the number of neighbors and their status. In RandomCast, each node receives ATIMs and ATIM-ACKs during an ATIM window and, thus, knows who will stay awake in the following data transmission period. This is useful to improve the performance because a node can send a packet without announcement if it knows that the receiver is awake. For this to work, a node should keep awake even if it does not receive an ATIM-ACK for its ATIM frame. This is, in fact, required in the 802.11 standard [2].

- ODPM: Like RandomCast, in ODPM, each node should know the status of its neighbors such as power management mode (AM or PS). This way, a node can send a packet without a prior announcement if the receiver is known to be in AM. However, even if the receiver is in AM, nodes are not allowed to send a packet during an ATIM window; otherwise, it could disrupt other nodes' ATIM or ATIMACK frames. No overhearing is used for unicast packets in ODPM in order to save energy. This choice is made on behalf of ODPM, where nodes wake up more often than other schemes. It makes ODPM comparable to 802.11 PSM and RandomCast with respect to energy performance.

\section{Performance Evaluation}

\subsection{Simulation Testbed}

The performance of RandomCast is evaluated using ns-2 [1], which simulates node mobility, a realistic physical layer, radio network interfaces, and the DCF protocol. Since ns-2 does not support 802.11 PSM, we modified the simulator based on suggestions in [7]. Our evaluation is based on the simulation of 50 mobile nodes located in an area of $1500^{-} \times \leftarrow$ $300-\mathrm{m}^{2}$. The radio transmission range is assumed to be $250 \mathrm{~m}$, and the two-ray ground propagation channel is assumed with a data rate of $2 \mathrm{Mbps}$. The data traffic simulated is constant bit rate (CBR) traffic. Twenty nodes out of 50 generate CBR streams at the data rate of 0.2-2.5 256-byte data packets every second $\left(R_{p k t}\right)$. Random waypoint mobility model [12] is used in our experiments with a maximum node speed of $5 \mathrm{~m} / \mathrm{s}$ and a pause time $\left(T_{\text {pause }}\right)$ of $0-900$ seconds. With this mobility model, a node travels (at $5 \mathrm{~m} / \mathrm{s}$ ) toward a randomly selected destination in the network. After the node arrives at the destination, it pauses for the predetermined period of time ( $\left.T_{\text {pause }}\right)$ and travels toward another randomly selected destination. Simulation time is 900 seconds, and each simulation scenario is repeated 10 times to obtain steadystate performance metrics.

We compare four different schemes: 802.11, 802.11 PSM, ODPM, and RandomCast. 802.11 is unmodified IEEE 802.11 without PSM. As discussed in Section 2.2, ODPM [32] is one of the most competitive energy-efficient schemes developed for multihop networks. For ODPM, a node remains in AM for 5 seconds if it receives an RREP (RREP time-out). It remains in AM for 2 seconds if it receives a data packet or it is a source or a destination node (Data time-out). These values are suggested in the original paper [32]. RandomCast uses no/unconditional/randomized overhearing depending on the packet type as explained in Section 3. We additionally evaluate RCAST, which employs randomized overhearing like RandomCast but not randomized rebroadcast. This is introduced to see the additional performance enhancement due to randomized rebroadcast.

ATIM window size and the beacon interval are set to 0.02 and 0.4 seconds in ODPM as suggested in its original paper [32]. On the contrary, they are 0.05 and 0.25 seconds in PSM and RandomCast as suggested in [29]. Since nodes are allowed to send packets without prior announcements in ODPM, they require a smaller ATIM window than in 802.11 PSM and RandomCast. Nonetheless, considering the relative overhead due to ATIM windows, ODPM is advantageous in terms of energy consumption. However, our simulation results show the opposite, which tells the superiority of the proposed RandomCast protocol. Table 1 summarizes protocol behaviors of the four schemes with their expected performance.

\subsection{Performance Metrics}

Performance metrics we have used in our experiments are energy consumption, energy goodput, packet delivery ratio (PDR), and packet delay. Energy consumption is measured at the radio layer during the simulation based on the specification of IEEE 802.11-compliant WaveLAN-II [14] from Lucent. The power consumption varies from $0.013 \mathrm{Watt}$ in a low-power sleep state to $0.83,1.0$, and 1.4 Watt in idle listening, receiving, and transmitting states, respectively, [10]. The instantaneous power is multiplied by the time duration to obtain energy consumption. In order to examine the performance trade-offs, a combined metric, called energy goodput (Kbytes/Joule), has been used in this paper. It measures the number of bytes delivered successfully per unit energy. 
TABLE 1

Protocol Behavior of Four Schemes

\begin{tabular}{|c|c|c|}
\hline Scheme & Behavior & Expected Performance \\
\hline 802.11 & $\begin{array}{l}\text { Does not incorporate PSM and nodes are always awake. } \\
\text { Thus, packets are transmitted immediately whenever they } \\
\text { are ready. }\end{array}$ & Best PDR and delay, but consumes the most energy. \\
\hline 802.11 PSM & $\begin{array}{l}\text { Nodes incorporate PSM, and overhear unconditionally. Packets } \\
\text { are deferred until it is announced in the next beacon interval. }\end{array}$ & $\begin{array}{l}\text { Saves energy compared to } 802.11 \text {, but consumes more energy than } \\
\text { necessary due to unconditional overhearing and unconditional } \\
\text { rebroadcasts. }\end{array}$ \\
\hline ODPM & $\begin{array}{l}\text { Nodes remain in AM for a pre-determined period of time } \\
\text { when they receive an RREP or a data packet or they are the } \\
\text { source or destination node. Packets intended for an AM node } \\
\text { are transmitted immediately. Packets intended for a PS node } \\
\text { are deferred. }\end{array}$ & $\begin{array}{l}\text { Less packet delay than RandomCast because some packets are } \\
\text { transmitted immediately. Higher energy cost than RandomCast } \\
\text { because some nodes remain in AM for an extended period } \\
\text { of time. }\end{array}$ \\
\hline RandomCast & $\begin{array}{l}\text { All nodes consistently operate in the PS mode and the level } \\
\text { of overhearing and rebroadcast is controlled. Packets are } \\
\text { deferred until it is announced in the next beacon interval. }\end{array}$ & $\begin{array}{l}\text { Less energy than ODPM. } \\
\text { No switches between AM and PS mode. }\end{array}$ \\
\hline
\end{tabular}

\subsection{Simulation Results}

Fig. 6 shows PDR, the average energy consumption per node, and energy goodputs for the five different schemes mentioned above with varying packet injection rate $(0.2-$ 2.5 packets/second). Fig. 6a shows that all five schemes deliver more than 90 percent of packets successfully under the low-traffic condition simulated. In the high packet injection rate, both 802.11 and ODPM show a higher PDR than 802.11 PSM, RCAST, and RandomCast because all (802.11) or more (ODPM) nodes are in AM and participate in the packet transmission. On the other hand, 802.11 and ODPM consume more energy than RCAST and RandomCast as shown in Fig. 6b. Fig. 6c compares the energy goodput. RandomCast achieves the best energy goodput performance regardless of the packet injection rate. In particular, under the low-traffic condition, the energy goodput of RandomCast is 2-2.5 times higher than 802.11. In comparison to

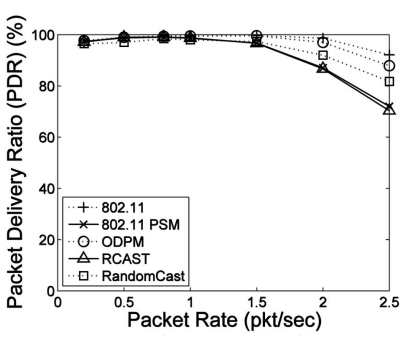

(a)

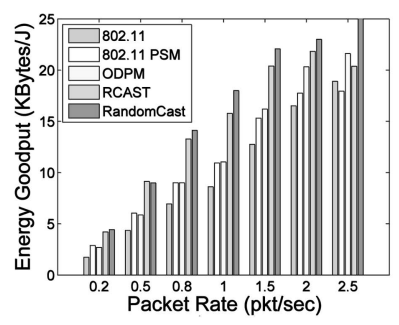

(c)

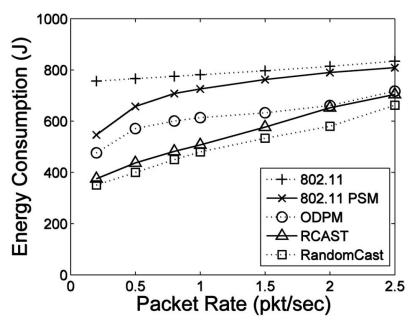

(b)

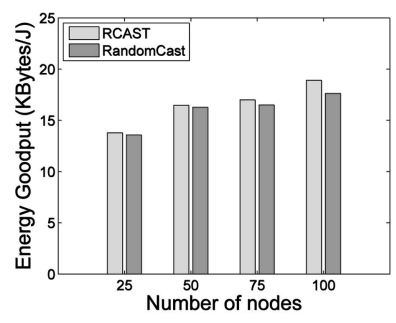

(d)
Fig. 6. Performance comparison. (As a reference, if a node stays idle for the entire 900 seconds, it consumes $900 \times 4.83=747$ - Joules. RCAST employs randomized overhearing but not the randomized rebroadcast.) (a) Packet delivery ratio (percent), (b) average energy consumption per node, (c) energy goodput (Kbytes/Joule), and (d) energy goodput versus number of nodes.
802.11 PSM and ODPM, energy goodput is improved by 30-64 percent and 13-63 percent, respectively.

It is important to note the performance difference between RCAST and RandomCast. RandomCast achieves a higher PDR, particularly when packet rate is high as shown in Fig. 6a. This is because of the lower network traffic due to broadcast packets with RandomCast. In addition, it achieves lower energy consumption as in Fig. 6b. Overall, its energy goodput is as much as 23 percent better than RCAST. It is noted that the additional energy savings due to randomized rebroadcast could be even larger when node mobility or node density is high. Fig. 6d compares RCAST and RandomCast with varying number of nodes. The performance gap is not dramatic, but we can observe a trend that verifies our argument. For example, when the number of nodes in the network is 100, RandomCast achieves 8 percent higher energy goodput.

Per-node energy consumption in Fig. $6 \mathrm{~b}$ has been redrawn in more detail in Fig. 7. Total energy consumption is broken down into transmission $(\mathrm{Tx})$, reception/overhearing (Rx), idle, and sleep energy. Figs. 7a, 7b, 7c, and 7d correspond to 802.11, 802.11 PSM, ODPM, and RandomCast, respectively. From the figures, we made the following interesting observations:

- First, energy consumption due to transmission and sleep is negligible. It represents only 0.3-3.0 percent (Tx) and 0.0-1.6 percent (sleep) of total energy consumption. Sleep energy is negligible because a node consumes two orders of smaller energy when it sleeps as discussed in Section 1.

- Second, energy consumption due to idle is in general the largest. This is the case with 802.11 as shown in Fig. 7a. In particular, when the packet injection rate is low, nodes consume most of their energy in an idle state. Therefore, to save energy, nodes should switch to a sleep state as much as possible while maintaining a good network performance. This is what RandomCast does. Idle energy is the smallest and it doesn't vary much with different packet rates as drawn in Fig. $7 \mathrm{~d}$. At the packet rate of 0.2 , RandomCast consumes 109 percent, 47 percent, and 27 percent less idle energy than 802.11, 802.11 PSM, and ODPM, respectively. This holds true for higher 


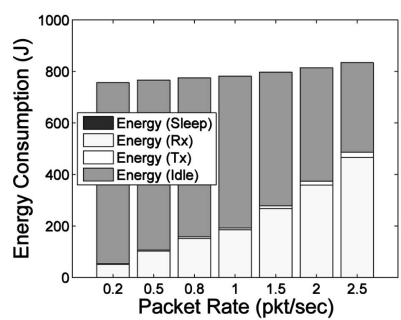

(a)

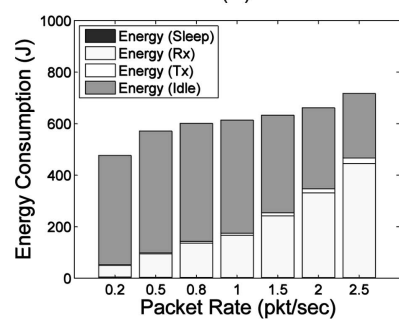

(c)

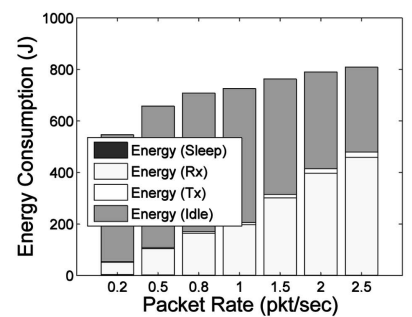

(b)

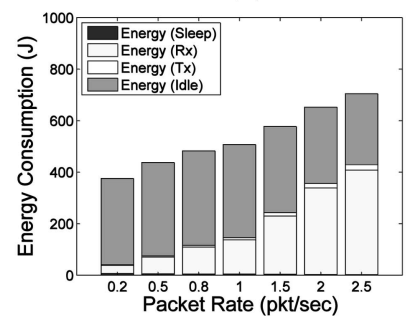

(d)

Fig. 7. Average energy consumption per node (energy consumption due to transmission $(\mathrm{Tx})$, reception/overhearing $(\mathrm{Rx})$, idle, and sleep is shown). (a) 802.11, (b) 802.11 PSM, (c) ODPM, and (d) RandomCast.

traffic condition except the packet rate of 2.5, where ODPM consumes less idle energy than RandomCast although the difference is just 24.8 Joules as shown in Figs. $7 \mathrm{c}$ and $7 \mathrm{~d}$. However, at that packet rate, ODPM consumes 37.6 Joules more Rx energy than RandomCast, resulting in more total energy consumption.

- Third, it is noted that RandomCast exhibits a relatively consistent idle energy regardless the traffic. Across the packet injection rate of 0.2-2.5, the maximum and the minimum idle energy differs 23.3 percent, which should be contrasted to 102.0 percent, 50.0 percent, and 69.6 percent with 802.11, 802.11 PSM, and ODPM, respectively. It is due to the judicious power management of RandomCast, and nodes are put to sleep better when there is no traffic to participate.

- Fourth, energy consumption due to reception/overhearing $(\mathrm{Rx})$ increases with traffic. As shown in Fig. 7, the Rx energy follows a similar pattern regardless the algorithm used. However, RandomCast exhibits the smallest $\mathrm{Rx}$ energy. It marks 14.9 percent, 10.9 percent, and 9.3 percent less $\mathrm{Rx}$ energy than the other three protocols at the packet rate of 0.2. The reduction becomes 61.0 percent, 50.3 percent, and 41.5 percent when the packet rate is 2.5. The benefit of RandomCast comes from a lower Rx energy when packet rate is high and it comes from a lower idle energy when packet rate is low.

While RandomCast is advantageous in terms of total energy, it doesn't necessarily bring in a balanced energy consumption across the nodes in the network. It is of primary interest because it affects the network lifetime as highconsuming nodes exhaust their batteries deteriorating the network connectivity in a MANET. To see the energy balance, Fig. 8 shows the energy consumption of all 50 nodes drawn in an increasing order of energy consumption. Figs. 8a and 8b show the results in static scenario (pause time of 900 seconds),

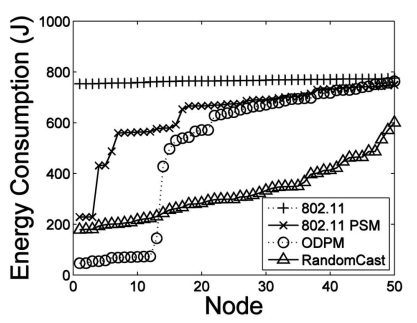

(a)

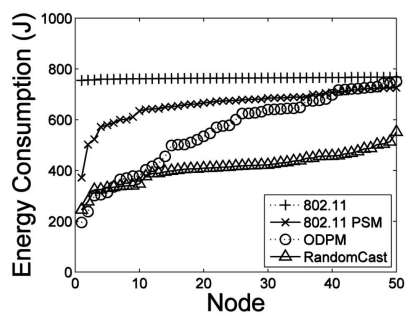

(c)

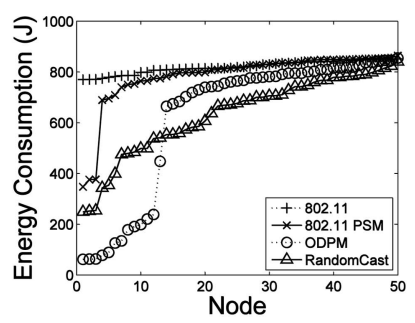

(b)

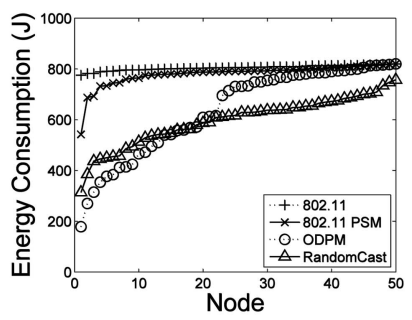

(d)
Fig. 8. Energy consumption of all 50 nodes (nodes are ordered in an increasing order of energy consumption for a better presentation). (a) $R_{p k t \psi}=4.5, T_{\text {pause } \psi}=900$, (b) $R_{p k t \psi}=2.0, T_{\text {pause } \psi}=900$, (c) $R_{p k t \psi}=4.5$, $T_{\text {pause }}=4$, and (d) $R_{\text {pkt }}=2.0, T_{\text {pause } \psi}=4$.

while Figs. $8 \mathrm{c}$ and $8 \mathrm{~d}$ show the results in mobile scenario (pause time of 0 second). Figs. 8a and 8c simulate low-traffic condition ( 0.5 packets/second) and Figs. $8 \mathrm{~b}$ and $8 \mathrm{~d}$ simulate higher traffic scenario (2.0 packets/second). In all the figures, 802.11 consumes the maximum energy since nodes keep awake during the entire period of simulation time (at least 0.83-Watt- $\times 900$-seconds $-=$ 747-Joules $)$.

Except 802.11, RandomCast outperforms the others with respect to energy balance, which is more significant in a static scenario as shown in Figs. 8a and 8b. In the two figures, ODPM exhibits two groups of nodes: high and low energyconsuming node groups. The former includes source and destination nodes. As explained in Section 4.1, they should keep awake (in AM) during the 900 seconds because the interpacket interval ( 2 seconds in Fig. $8 \mathrm{a}$ and 0.5 second in Fig. $8 \mathrm{~b}$ ) is not less than the predefined Data time-out (2 seconds). All intermediate nodes between a source and a destination belong to the same high energy-consuming group due to the same reason. Other nodes belong to the latter group. They would not be bothered and wake up only during the ATIM windows consuming less energy ( 0.83 Watt $\times 45$-seconds -0.013 -Watt $-\times 855$-seconds $=448.465$-Joules $)^{4}$ as shown in Figs. 8a and 8b. This division doesn't exist with RandomCast because nodes in the vicinity of active communication overhear probabilistically. More nodes participate in the communication leading to a better energy balance.

On the other hand, the division disappears even in ODPM in a mobile scenario as drawn in Figs. 8c and 8d. However, RandomCast still exhibits a better balance than ODPM. It is noted from Fig. 8 that in ODPM, some nodes consume an extremely small amount of energy, which is not observed in RandomCast and 802.11 PSM. This is because ODPM uses a smaller ATIM window size $(0.02$ second in 0.4 second

4. In our experiment, beacon interval is 0.4 second and ATIM window size is 0.02 second in ODPM. Thus, there are 2,250 beacon intervals during the 900 seconds of simulation time. Nodes in the latter group are in AM for $2,250-\times 4.02-=45$ - seconds and in PS mode for the rest 855 seconds. 


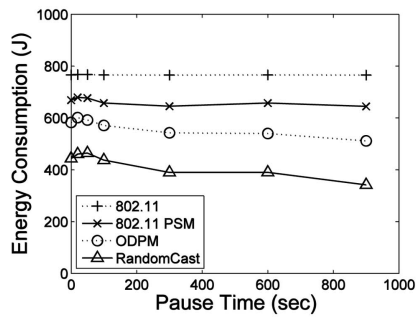

(a)

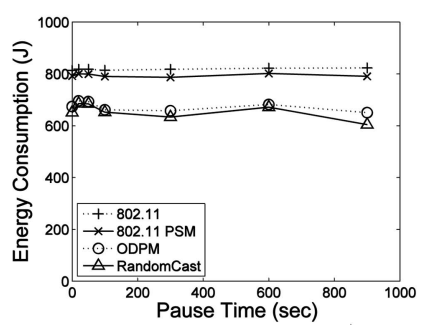

(c)

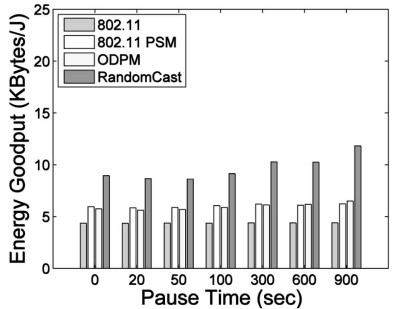

(b)

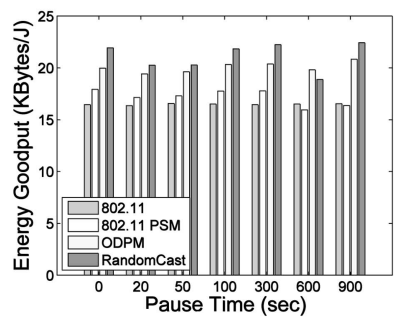

(d)
Fig. 9. Energy performance versus mobility. (a) Average energy consumption per node $\left(R_{p k t \psi}=4.5\right)$, (b) energy goodput (Kbytes/Joule) $\left(R_{p k t \psi}=\theta .5\right)$, (c) average energy consumption per node $\left(R_{p k t \psi}=2.0\right)$, and (d) energy goodput (Kbytes/Joule) $\left(R_{p k t \psi}=2.0\right)$.

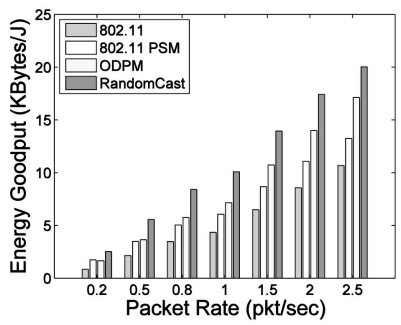

(a)

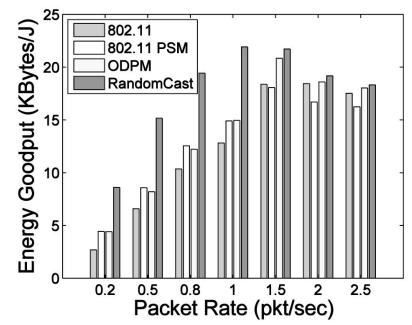

(b)
Fig. 10. Energy goodput (Kbytes/Joules) versus number of CBR connections (experiment result with $20 \mathrm{CBR}$ connections is in Fig. 7c). (a) 10 CBR connections. (b) 30 CBR connections.

beacon interval or 5 percent $)$ than RandomCast ( 0.05 second in 0.25 second beacon interval or 20 percent) as discussed in Section 4.1. If they use the same ATIM window and beacon interval, the performance superiority of RandomCast over ODPM will be even more significant.

Fig. 9 shows the average per-node energy consumption and energy goodput versus mobility. In Figs. 9a and 9b, when packet injection rate is 0.5 packets/second, ODPM shows as much as 40.8 percent more energy consumption and as much as 80.2 percent less energy goodput than RandomCast. The gap is larger with less mobility. Under a high traffic scenario shown in Figs. 9c and 9d, the difference in energy performance between ODPM and RandomCast decreases. However, RandomCast still shows less energy consumption and more energy goodput than ODPM as shown in the figure.

Fig. 10 shows energy goodputs with different number of CBR traffic streams. With both 10 and 30 CBR connections, RandomCast consistently shows the highest energy goodput. With 10 CBR connections, it achieves 16.9-53.5 percent higher energy goodput than ODPM as shown in Fig. 10a. On the other hand, the performance advantage reduces to 0.29.5 percent with $30 \mathrm{CBR}$ connections in Fig. 10b. Note that in Fig. 10b, energy goodput saturates when the packet rate

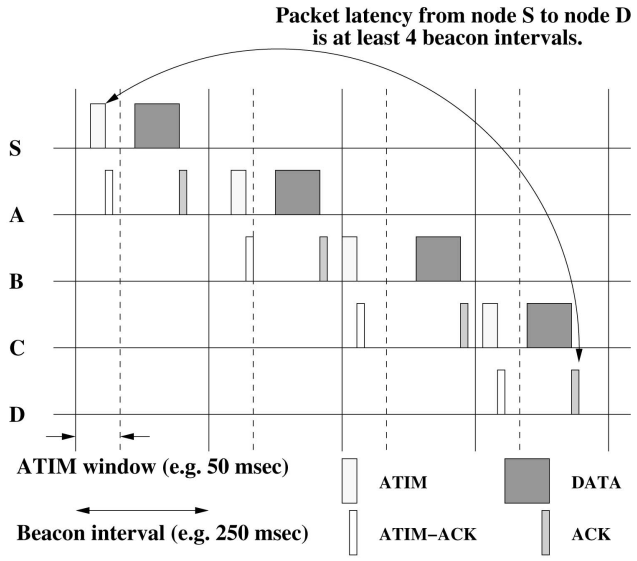

(a)

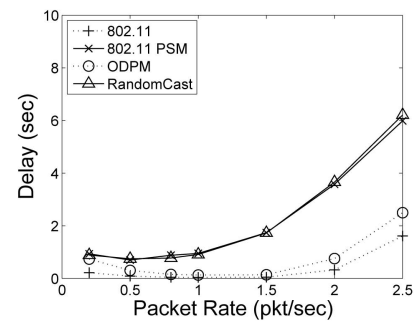

(b)

Fig. 11. Delay performance $\left(T_{\text {pause }}=400\right)$. (a) Multihop delivery in PSMbased protocols and (b) average packet latency.

exceeds 1.5 packets per second, where the traffic is simply higher than what the network can tolerate.

In short, RandomCast performs on par with other schemes in terms of PDR but achieves a significant energy saving as well as a better energy balance in comparison to existing schemes. The benefit of RandomCast is significant when traffic is light. This is because nodes stay in low-power sleep state more intelligently in RandomCast. It consumes less energy at high traffic condition as well, but the benefit in this case comes from less Rx energy. This is credited to more judicious overhearing decisions than other schemes.

\section{Related Work}

This paper concentrates on energy performance of 802.11 PSM-based MAC protocols. However, packet latency is at a disadvantage in any PSM-based protocols because a packet is announced before an actual delivery and the announcement can only be made one hop at a time in each beacon interval. This is shown in Fig. 11a. For a 4-hop routing path $(S \psi \rightarrow \uplus)$, the packet latency is at least 4 beacon intervals or 1.0 second when beacon interval is 0.25 second. Fig. $11 b$ shows the average packet latency observed during the simulation under the simulation scenario explained in Section 4.1. 802.11 and ODPM show the smallest packet latency. This is because all (802.11) or some (ODPM) nodes transmit data packets immediately without waiting for the next beacon interval as discussed in Section 3.6. In both 802.11 PSM and RandomCast, each node must wait a beacon interval ( 0.25 second) for each hop, resulting in an extended latency. In addition, each link breakage extends the packet latency because it requires the delivery of RERR 
to the source as well as the broadcast of RREQ toward the destination, each of which takes a few beacon intervals. This happens more in high mobility or high traffic condition as shown in Fig. 11b.

The aforementioned latency problem in PSM-based protocols has been addressed in some recent research work. $\mathrm{Hu}$ and Hou considered a mechanism that does not trade off end-to-end performance without compromising energy performance [10]. They observed that a major source for packet delay in 802.11 PSM is wake-up latency; a packet can only be traversed one hop in one beacon interval. Even worse, any intermediate node has to wake up twice for packet forwarding-one to receive and the other one to forward [10]. Their proposed mechanism, called LinkIndexed Statistical traffic Prediction (LISP), introduces pseudo- $A C K$ packet. When a sender and a receiver exchange ATIM and ATIM-ACK in an ATIM window, the next downstream node can send pseudo-ACK packet to inform that it can forward the packet during the subsequent beacon interval [10]. A critic of this scheme is that it requires inconsistent modification to the 802.11 standard because ATIM and ATIM-ACK must contain information about the next downstream node.

Dorsey and Siewiorek discussed a similar problem with a special focus on route discovery procedure [7]. For the same reason stated above, the latency for a route discovery is several orders of magnitude greater than that experienced without PSM. They proposed Fast wakeup mechanism, where a received ATIM for a broadcast packet triggers a priority transmission of the ATIM for rebroadcast of the same broadcast packet [7]. The consequence is that broadcast packets such as RREQs may travel more than a hop in a beacon interval.

Although the aforementioned schemes can be integrated with RandomCast to improve the delay performance, we do not pursue this in this paper to clearly see the performance changes contributed by the randomized overhearing and rebroadcasts in RandomCast.

\section{Conclusions}

Little effort has been devoted to integrate 802.11 PSM with a multihop routing protocol such as DSR. This study addresses this important problem and suggests an efficient solution based on RandomCast. The key observation is that unconditional overhearing, which is taken for granted without PSM, is not freely available with PSM. In RandomCast, when a packet is transmitted, nodes in the proximity should decide whether or not to overhear it considering the trade-offs between energy efficiency and routing efficiency. Routing efficiency comes into picture because overhearing is an important tool to gather route information in DSR. Similarly, we explored the use of RandomCast for broadcast messages in order to avoid redundant rebroadcasts and thus save additional energy.

This paper compares the performance of RandomCast with four other schemes in terms of PDR, energy consumption, energy goodput, and energy balance through simulation. Our results indicate that RandomCast significantly outperforms ODPM (as much as 30 percent less energy), which is the most competitive scheme developed for multihop networks employing on-demand routing algorithms, without significantly deteriorating the general network performance such as PDR. RandomCast also improves energy goodput by as much as 56 percent, that is, an integrated measure of energy and PDR. The performance results indicate that the proposed scheme is quite adaptive for energy-efficient communication in MANETs. In particular, applications without stringent timing constraints can benefit from the RandomCast scheme in terms of power conservation.

RandomCast opens many interesting directions of research to pursue. First, this paper identifies four factors that must be considered for the overhearing/rebroadcast decision. These are sender ID, number of neighbors, mobility, and remaining battery energy. We implemented the RandomCast scheme using only the second factor (number of neighbors), but we plan to investigate the effect of other three factors (sender ID, mobility, and remaining battery energy) for making the decision. Since these factors increase the corresponding overheads, we also need to assess their trade-offs. In particular, sender ID is the most compelling idea and can be implemented easily with a simple hashing function. Remaining battery energy will play an important factor if energy balance is critically important. We plan to incorporate the concept of RandomCast with other routing protocols.

\section{ACKNOWLEDGMENTS}

This research was supported in part by US National Science Foundation grants CNS-0509251, CCF-0429631, CNS-0831673, and CNS-0831853, and the World Class University (WCU) program through the Korea Science and Engineering Foundation funded by the Ministry of Education, Science, and Technology (Project No. R31-2008000-01000-0). This work was published in part at the 25th International Conference on Distributed Computing and Systems (ICDCS) 2005 [19].

\section{REFERENCES}

[1] VINT Project, The UCB/LBNL/VINT Network Simulator-ns (Version 2), http://www.isi.edu/nsnam/ns, 2009.

[2] IEEE Std 802.11-1999, Local and Metropolitan Area Network, Specific Requirements, Part 11: Wireless LAN Medium Access Control (MAC) and Physical Layer (PHY) Specifications, IEEE, http://standards. ieee.org/getieee802/download/802-11-1999.pdf, 1999.

[3] S. Agarwal, S.V. Krishnamurthy, R.H. Katz, and S.K. Dao, "Distributed Power Control in Ad-Hoc Wireless Networks," Proc. IEEE Int'l Symp. Personal, Indoor and Mobile Radio Comm. (PIMRC '01), pp. 59-66, 2001.

[4] R. Chandra, V. Ramasubramanian, and K.P. Birman, "Anonymous Gossip: Improving Multicast Reliability in Mobile Ad-Hoc Networks," Proc. IEEE Int'l Conf. Distributed Computing Systems (ICDCS '01), pp. 275-283, 2001.

[5] J.-H. Chang and L. Tassiulas, "Energy Conserving Routing in Wireless Ad-Hoc Networks," Proc. IEEE INFOCOM, pp. 22-31, 2000.

[6] B. Chen, K. Jamieson, R. Morris, and H. Balakrishnan, "Span: An Energy-Efficient Coordination Algorithm for Topology Maintenance in Ad Hoc Wireless Networks," Proc. ACM MobiCom, pp. 85-96, 2001.

[7] J. Dorsey and D. Siewiorek, "802.11 Power Management Extensions to Monarch ns," Technical Report CMU-CS-04-183, School of Computer Science, Carnegie Mellon Univ., Dec. 2004.

[8] J. Gomez, A.T. Campbell, N. Naghshineh, and C. Bisdikian, "Conserving Transmission Power in Wireless Ad Hoc Networks," Proc. Ninth IEEE Int'l Conf. Network Protocols (ICNP '01), pp. 24-34, 2001.

[9] Z.J. Hass, J.Y. Halpern, and L. Li, “Gossip-Based Ad Hoc Routing," Proc. IEEE INFOCOM, pp. 1707-1716, 2002. 
[10] C. Hu and J. Hou, "A Link-Indexed Statistical Traffic Prediction Approach to Improving IEEE 802.11 PSM," J. Ad Hoc Networks, vol. 3, no. 5, pp. 529-545, 2005.

[11] L. Huang and T.-H. Lai, "On the Scalability of IEEE 802.11 Ad Hoc Networks," Proc. ACM MobiHoc, pp. 173-182, 2002.

[12] D.B. Johnson and D.A. Maltz, "Dynamic Source Routing in Ad Hoc Wireless Networks," Mobile Computing, pp. 153-181, Kluwer, 1996.

[13] E.-S. Jung and N.H. Vaidya, "A Power Control MAC Protocol for Ad Hoc Networks," Proc. ACM MobiCom, pp. 36-47, 2002.

[14] A. Kamerman and L. Monteban, "WaveLAN II: A High Performance Wireless LAN for the Unlicensed Band," Bell Labs Technical J., vol. 2, pp. 118-133, Summer 1997.

[15] V. Kawadia and P. Kumar, "Power Control and Clustering in Ad Hoc Networks," Proc. IEEE INFOCOM, pp. 459-469, 2003.

[16] R. Kravets and P. Krishnan, "Power Management Techniques for Mobile Communication," Proc. ACM MobiCom, pp. 157-168, 1998.

[17] P. Kyasanur, R.R. Choudhury, and I. Gupta, "Smart Gossip: An Adaptive Gossip-Based Broadcasting Service for Sensor Networks," Proc. Second IEEE Int'l Conf. Mobile Ad Hoc and Sensor Systems (MASS '06), pp. 91-100, 2006.

[18] Z. Li and B. Li, "Probabilistic Power Management for Wireless Ad Hoc Networks," Mobile Networks and Applications, vol. 10, no. 5, pp. 771-782, 2005.

[19] S. Lim, C. Yu, and C. Das, "Rcast: A Randomized Communication Scheme for Improving Energy Efficiency in Mobile Ad Hoc Networks," Proc. 25th IEEE Int'l Conf. Distributed Computing Systems (ICDCS '05), pp. 123-132, 2005.

[20] M.K. Marina and S.R. Das, "Performance of Route Caching Strategies in Dynamic Source Routing," Proc. Int'l Workshop Wireless Networks and Mobile Computing (WNMC '01), pp. 425432, 2001.

[21] M. Miller, C. Sengul, and I. Gupta, "Exploring the Energy-Latency Tradeoff for Broadcasts in Energy-Saving Sensor Networks," Proc. IEEE Int'l Conf. Distributed Computing Systems (ICDCS '05), pp. 1726, 2005

[22] M. Sanchez, P. Manzoni, and Z.H. Hass, "Determination of Critical Transmission Range in Ad-Hoc Networks," Proc. Multiaccess, Mobility, and Teletraffic for Wireless Comm. Workshop (MMT '99), 1999.

[23] C. Sengul and R. Kravets, "Conserving Energy with On-Demand Topology Management," Proc. Second IEEE Int'l Conf. Mobile Ad Hoc and Sensor Systems (MASS '05), pp. 10-19, 2005.

[24] S. Singh, M. Woo, and C.S. Raghavendra, "Power-Aware Routing in Mobile Ad Hoc Networks," Proc. ACM MobiCom, pp. 181-190, Oct. 1998.

[25] Y.-C. Tseng, Y. Chen, and J. Sheu, "The Broadcast Storm Problem in a Mobile Ad Hoc Network," Proc. ACM MobiCom, pp. 151-162, 1999.

[26] Y.-C. Tseng, C.-S. Hsu, and T.-Y. Hsieh, "Power-Saving Protocol for IEEE 802.11-Based Multi-Hop Ad Hoc Networks," Proc. IEEE INFOCOM, pp. 200-209, 2002

[27] Y.-C. Tseng, S.-Y. Ni, Y.-S. Chen, and J.-P. Sheu, "The Broadcast Storm Problem in a Mobile Ad Hoc Network," Wireless Networks, vol. 8, nos. 2/3, pp. 153-167, 2002.

[28] M. Venkataraman and P. Gupta, "Stack Aware Architecture for Ad Hoc Networks," Internet draft, work in progress, 2004.

[29] H. Woesner, J. Ebert, M. Schlager, and A. Wolisz, "Power-Saving Mechanisms in Emerging Standards for Wireless LANs: The MAC Level Perspective," IEEE Personal Comm., vol. 5, no. 3, pp. 40-48, June 1998.

[30] K. Woo, C. Yu, H.Y. Youn, and B. Lee, "Non-Blocking, Localized Routing Algorithm for Balanced Energy Consumption in Mobile Ad Hoc Network," Proc. Ninth Int'l Symp. Modeling, Analysis and Simulation of Computer and Telecomm. Systems (MASCOTS '01), pp. 117-124, 2001.

[31] J. Wu, M. Gao, and I. Stojmenovic, "On Calculating Power-Aware Connected Dominating Sets for Efficient Routing in Ad Hoc Wireless Network," Proc. Int'l Conf. Parallel Processing (ICPP '01), pp. 346-356, 2001.

[32] R. Zheng and R. Kravets, "On-Demand Power Management for Ad Hoc Networks," Proc. IEEE INFOCOM, pp. 481-491, 2003.

Post-print standardized by MSL Academic Endeavors, the imprint of the Michael Schwartz Library at Cleveland State University, 2014 